\title{
En el quinto centenario de la muerte de Cisneros: breve semblanza de Demetrio Ducas, primer catedrático de griego de la Complutense
}

En estas fechas en que celebramos el quinto centenario de la muerte del cardenal Cisneros (1436-1517) resulta oportuno trazar una semblanza, aunque sea breve, de una figura mucho menos conocida, pero muy relevante para la historia de los estudios helénicos en la Universidad Complutense: Demetrio Ducas (ca. 1480-ca. 1527).

Corría el año de 1499 cuando el Cardenal fundaba la Universidad de Alcalá de Henares. Hacia 1513 llega a ella, llamado por Cisneros, Demetrio Ducas, un griego de Creta que había trabajado previamente en Venecia al lado del conocido tipógrafo Aldo Manuzio para preparar varias ediciones, entre ellas la de los Rhetores Graeci de 1508-1509 y la de los Moralia de Plutarco, también en 1509. La tarea a él encomendada por Cisneros era la de colaborar en la edición de la Biblia Políglota Complutense (en la que también colaboró el gran latinista Antonio de Nebrija), especialmente la supervisión de los textos del Nuevo Testamento incluidos en su tomo V, y encargarse de la recién creada cátedra de griego en dicha Universidad. Su labor no fue fácil porque en la Biblioteca del Colegio de S. Ildefonso apenas pudo encontrar libros griegos, lo que le animó a editar él mismo en 1514, con mucho esfuerzo y a sus propias expensas, varias obras griegas: un texto poético (Hero y Leandro, de Museo) y -sobre todo para el propósito que aquí nos interesa- una miscelánea para el aprendizaje del griego antiguo que suele citarse como Erotemata ("Cuestiones"), compuesta fundamentalmente por varios textos gramaticales de los eruditos bizantinos Manuel Crisoloras, Demetrio Calcóndila y Teodoro de Gaza, además de algunos otros opúsculos y una colección de sentencias, que reproducen en esencia una recopilación gramatical que el propio Aldo Manuzio había publicado dos años antes.

Estos Erotemata editados por Ducas, de los que se conserva un ejemplar en la Biblioteca Histórica Marqués de Valdecilla, de la Universidad Complutense de Madrid (signatura BH DER 140), tienen el indudable mérito de ser el primer Manual o Método para el aprendizaje del Griego antiguo editado en nuestro país. Su propósito, eminentemente docente, queda claro en la dedicatoria que el propio Ducas incluye en griego clásico al final de la edición y que dirige a los "estudiosos de la Universidad Complutense", así como las penalidades que al propio Ducas le acarreó su fatigosa elaboración (la traducción y destacados son nuestros):

"El cretense Demetrio Ducas saluda a los estudiosos de la Universidad Complutense:

Tras llegar a España, llamado por el reverendísimo Cardenal de España ciertamente por mi conocimiento de la lengua griega, y habiendo hallado una gran penuria 
de libros griegos o, mejor dicho, una completa ausencia de ellos, yo, tras imprimir como mejor me era posible algunos textos gramaticales y poéticos con los caracteres que me encontré aquí, os los he ofrecido como regalo a vosotros. Sin que nadie colaborara conmigo en los elevados gastos de impresión ni en las penalidades de la corrección, yo solo, a duras penas, me basté, leyendo cada día, copiando y corrigiendo a la vez. A vosotros, pues, os toca recibir benévolamente lo trabajado por mí con sudor, falta de sueño y dinero, y quedarme agradecidos. Yo, por mi parte, reconoceré haber recibido vuestro agradecimiento si, cultivando su estudio, llegáis a haceros expertos en la cultura griega. Saludos".

El texto es elocuente y no precisa de mayor comentario, especialmente para todos los que amamos las Clásicas y somos, o nos sentimos, "complutenses". Sí cabe recordar que esos "caracteres griegos" que Ducas halló en Alcalá fueron los empleados en la edición del Nuevo Testamento de la Biblia Políglota, preparados por el impresor Guillén de Brocar, a los que Ducas, que antes había trabajado en la espléndida imprenta aldina de Venecia, no estaba acostumbrado, y que seguramente fueron fuente de algunas erratas, incluso en esa misma dedicatoria.

Ducas, una figura que, según una de sus mejores conocedoras, Teresa Martínez Manzano, sigue siendo nebulosa y enigmática - no conservamos ninguna imagen suya, pero sí, posiblemente, algunos textos manuscritos-, abandonó Alcalá en 1518, después de morir Cisneros, para volver a Italia. Después de 1527 su rastro se pierde. Su paso por Alcalá fue relativamente fugaz, pero muy fructífero para la implantación de los estudios helénicos en nuestro país y con honda influencia en algunos humanistas españoles contemporáneos, especialmente en Hernán Núñez el Pinciano, quien seguramente se benefició de la consulta de la biblioteca privada de Ducas y de la copia de algunos de sus manuscritos.

El resto de la historia nos es más conocida. El 29 de octubre de 1836 el entonces Ministerio de la Gobernación publica la "Real orden trasladando a Madrid la Universidad de Alcalá con las demás disposiciones a ello concernientes" y la Universidad Complutense de Madrid se convierte en sucesora de la antigua Complutense de Alcalá. Son, por tanto, más de 500 años de historia de estudios helénicos en la Complutense, tanto de la antigua de Alcalá como de la más reciente de Madrid, que han tenido a la sección de Clásicas como núcleo fundacional y a Demetrio Ducas como su primer catedrático de Griego, un hecho que en este año en que se recuerda el quinto centenario de la muerte de su fundador, el cardenal Cisneros, quizá sea oportuno recordar. Por ello, en honor de Ducas se le dedicó el Congreso sobre docencia en Clásicas en la Comunidad de Madrid que en enero de 2017 se celebró en la Facultad de Filología de la Universidad Complutense de Madrid, y por ello también el Departamento de Filología Griega y Lingüística Indoeuropea acordó de manera unánime, en reunión reciente, nombrar a Demetrio Ducas "profesor protoemérito" a título honorífico, para que su nombre sea recordado como fundador y primer profesor del Departamento.

Felipe G. Hernández Muñoz Universidad Complutense de Madrid 


\section{Drmetl}

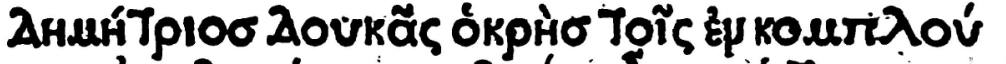

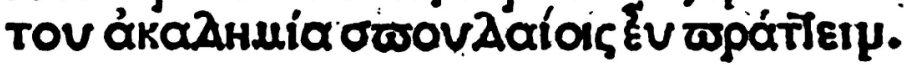

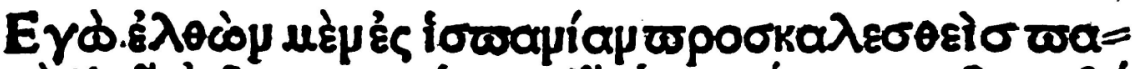

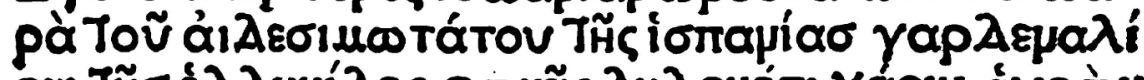

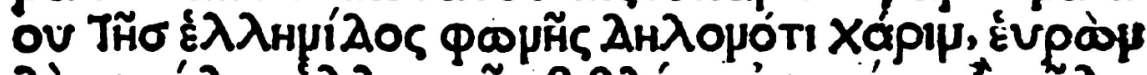

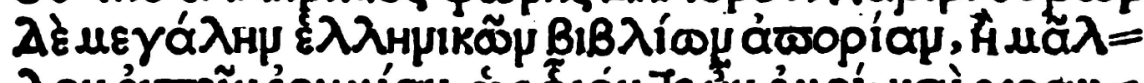

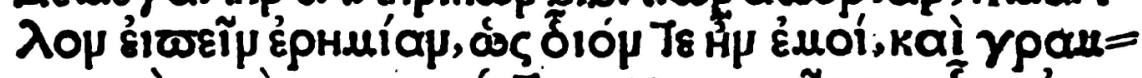

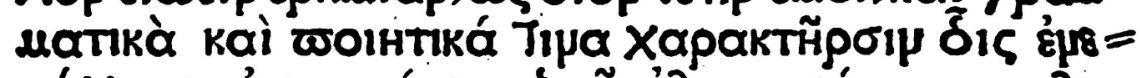

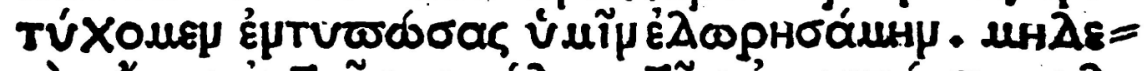

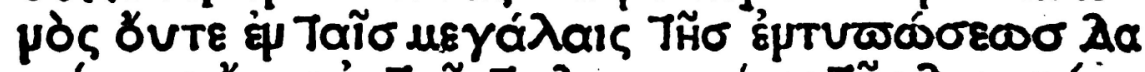

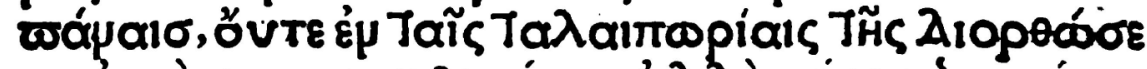

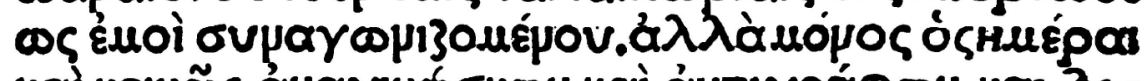

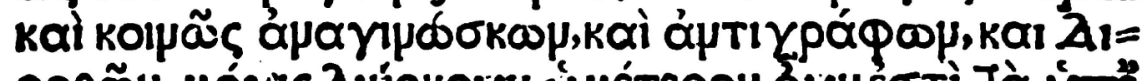

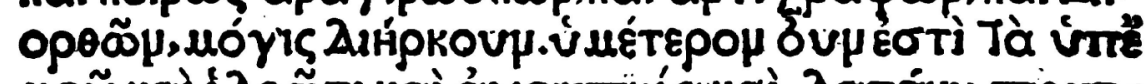

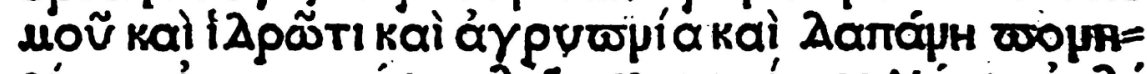

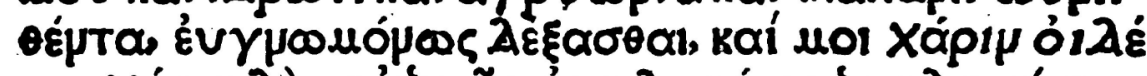

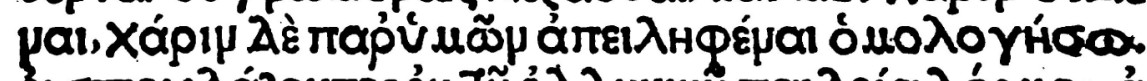

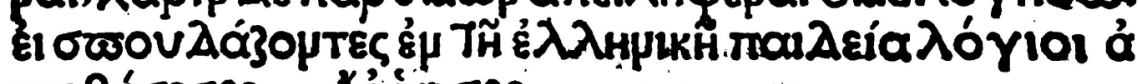

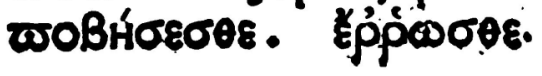

Figura 1. Dedicatoria final de los Erotemata, Biblioteca Histórica UCM. 


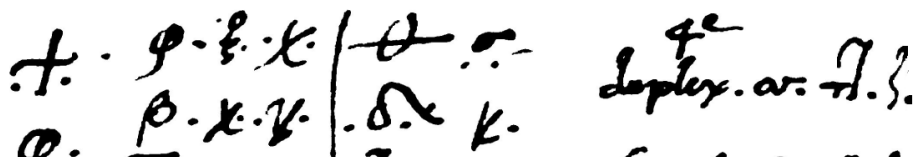

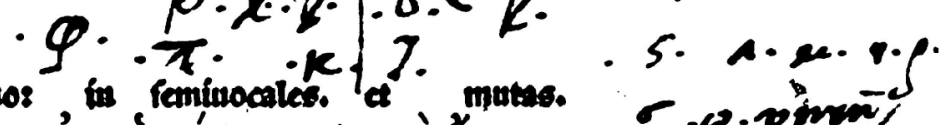

Quot remiluccales? acto.

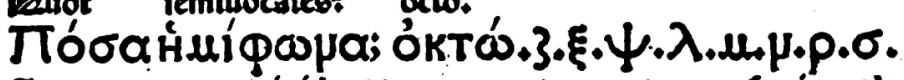

Jn quot sinidunterr octo remiluocalos? in

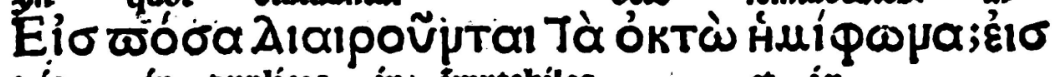

tria. in ouplices. in imurabiles. at in

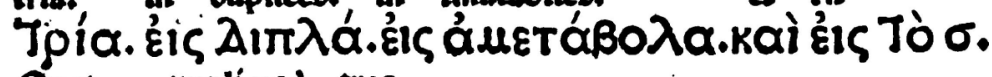

Ruos suplices? tres,

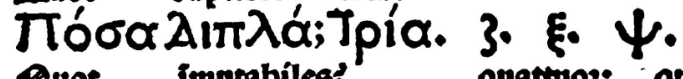

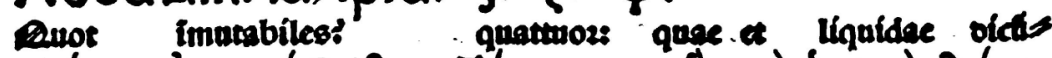

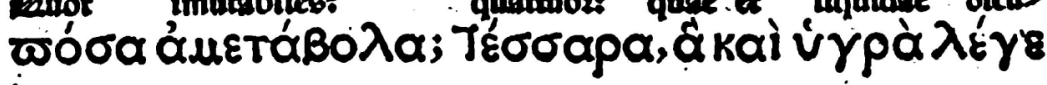
tur.

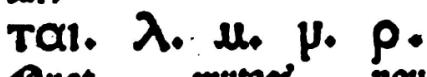

Buot muraet noum.

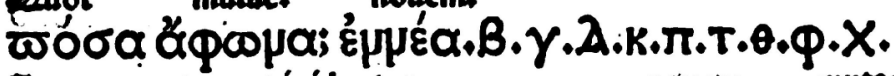

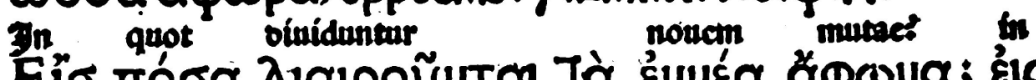

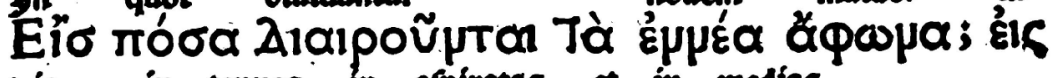
erria. in tenues, in aspiratess. et in medias

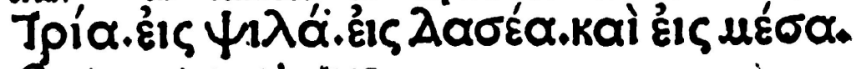

2unot. tenues? tres.

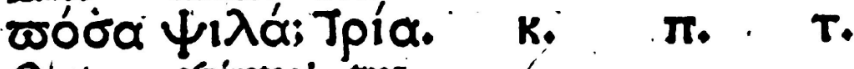

Dhot ápiratace tres.

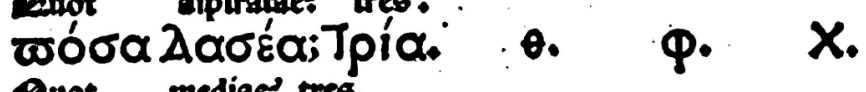

pót medíciat tres.

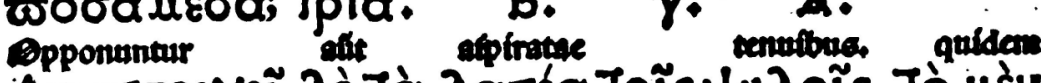

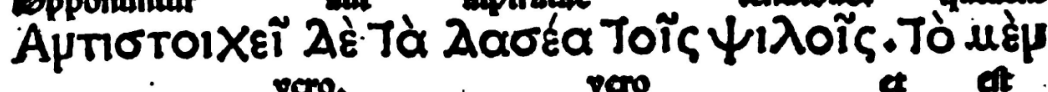

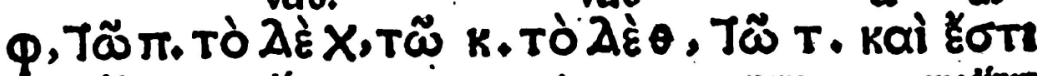

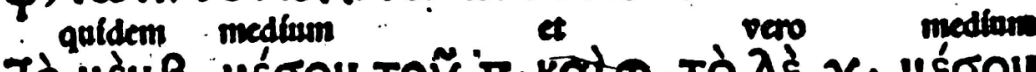

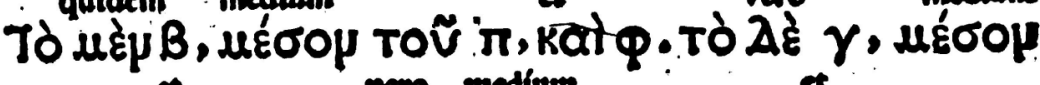

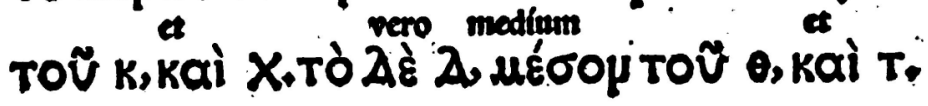

Figura 2. Comienzo de los Erotemata (sección de fonética griega), Biblioteca Histórica UCM. 
we vedingtionc

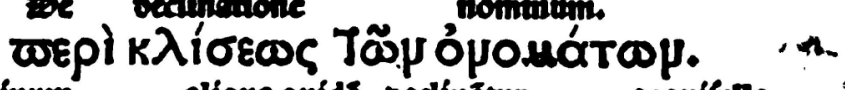
Dominum

aliquaquide oeclinatur

sequifithe

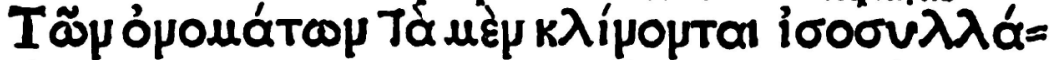
bice. it seneas. sencese. slíq vero plurifpl

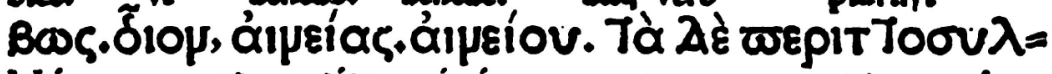
lebice. ot qiax. aincis. quere vocetur, beace

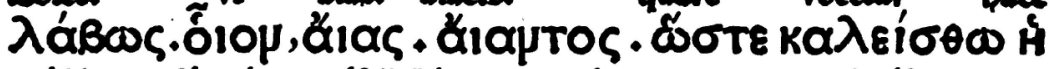
quidz: vedinatiogacquifillabica. becevero: plurfifi

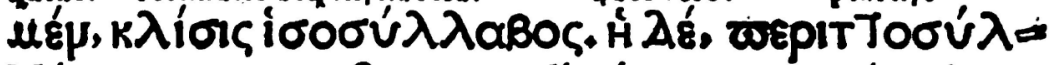
lablea. anot beclinationes nominum?

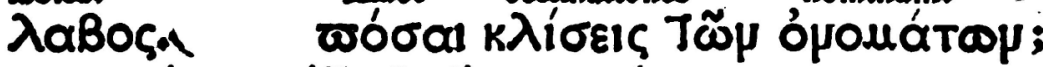
cecem. quinq quide fimplices: a incontractses quarum quar

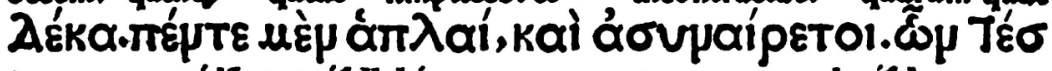
mo2 quidz sequifpllabicace ma vero plurift

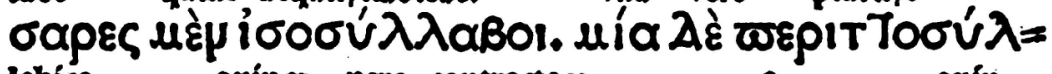
lebica. quinqs vero contractae:

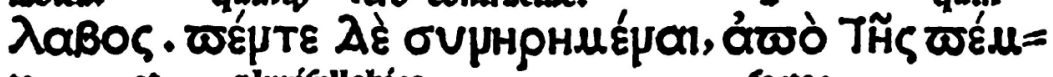
a et purfifyllabica

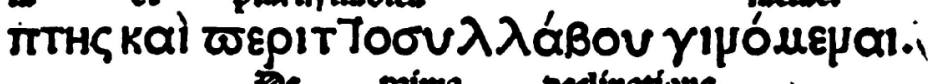

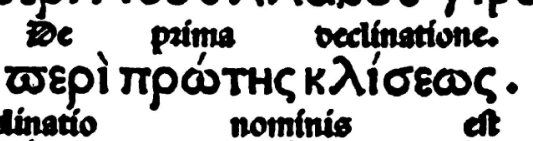

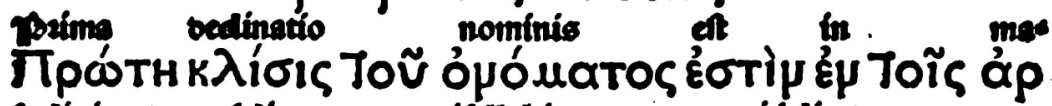
reulinis rolls sequifinlabica. videllicet, quo

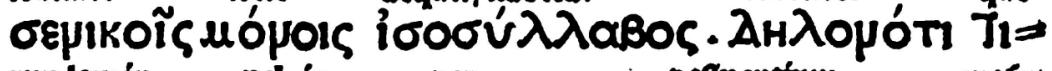
rundamin vel in per beflinentum, mercu

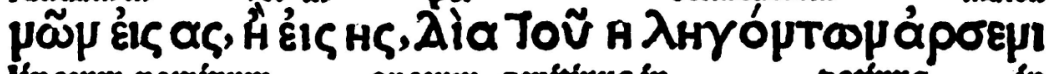
ilnomm nomtrum quosum gentifuus in

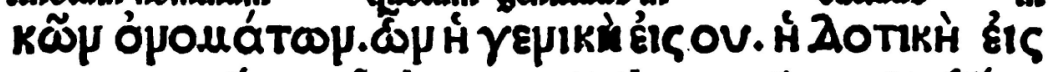
recti finalem vocalem. at ecculatures

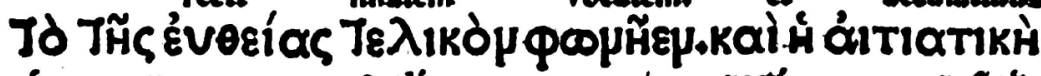

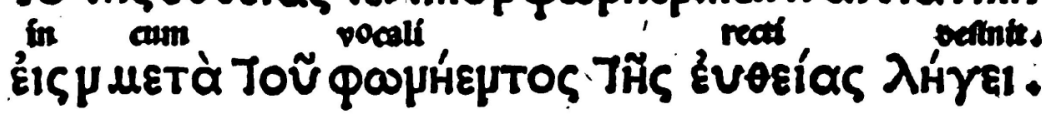

Figura 3. Erotemata, comienzo de la morfología nominal (primera declinación), Biblioteca Histórica UCM. 


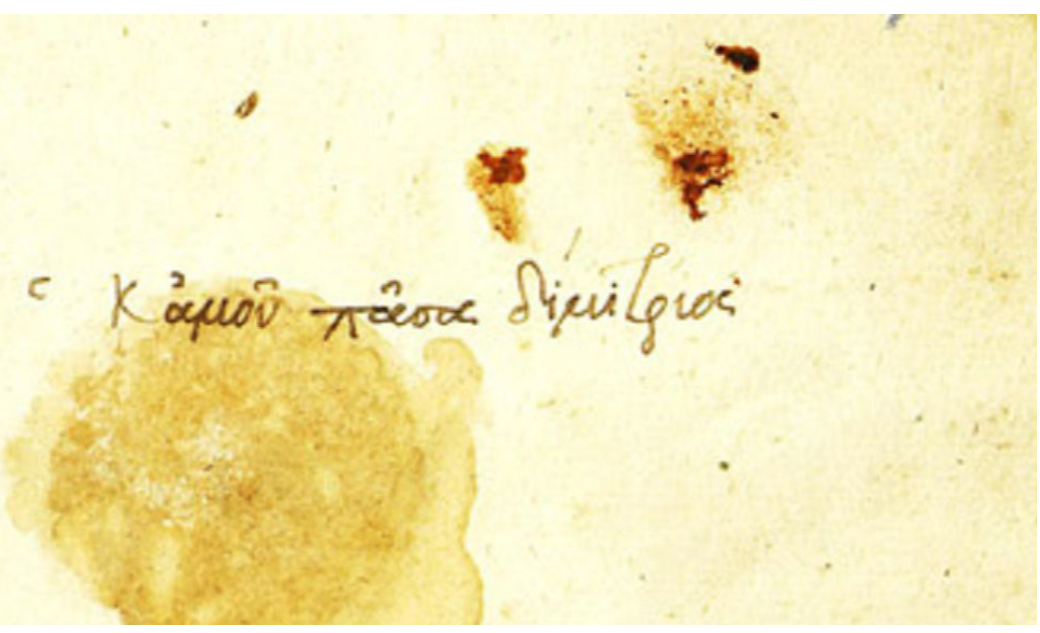

Figura 4. Probable "ex libris" de Demetrio Ducas en Salamanca, Incunable 157 (gentileza de Óscar Lilao, de la Universidad de Salamanca). 


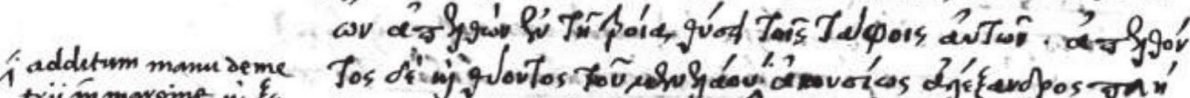

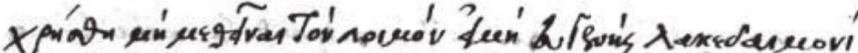

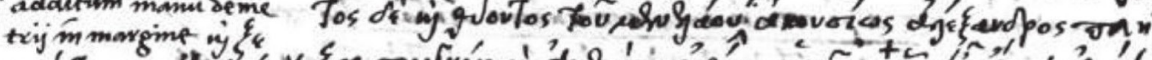

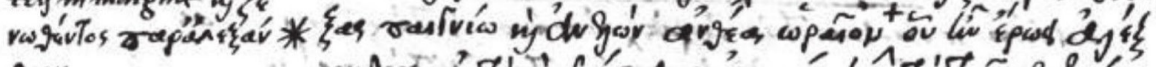

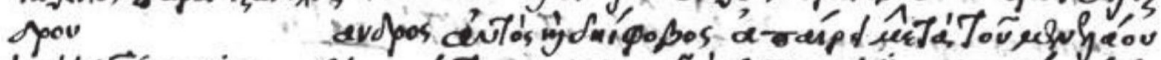

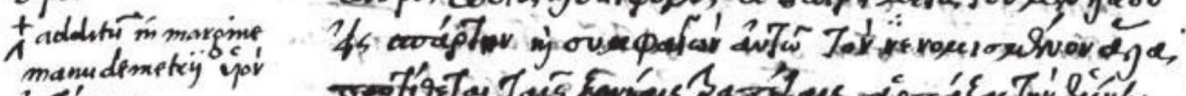
'av'Tinopos

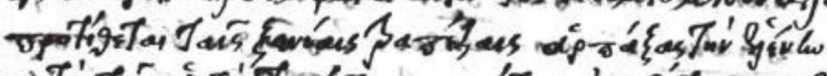

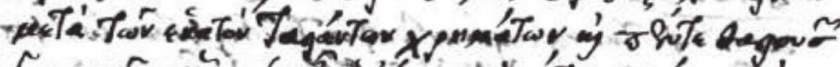

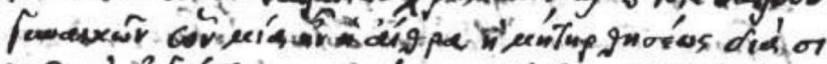

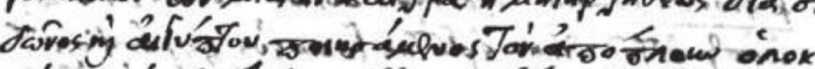

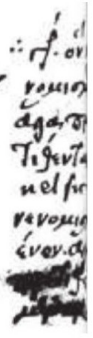

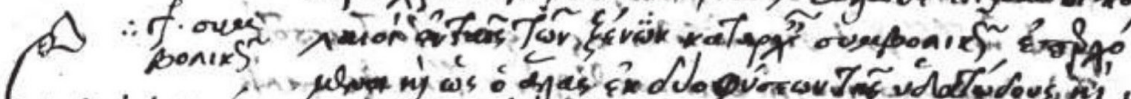

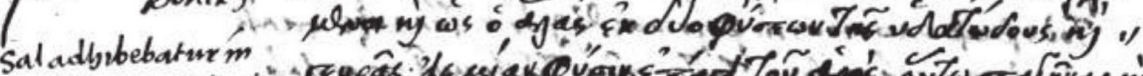

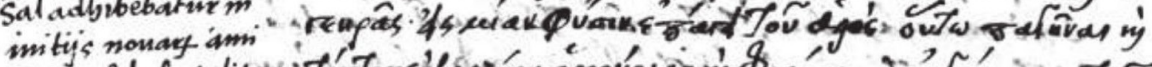

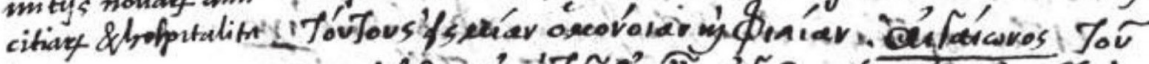

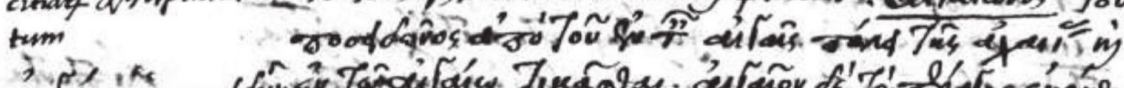

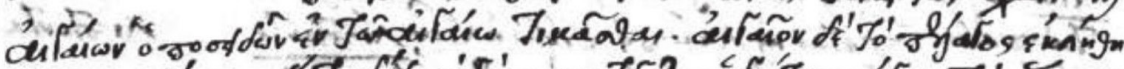

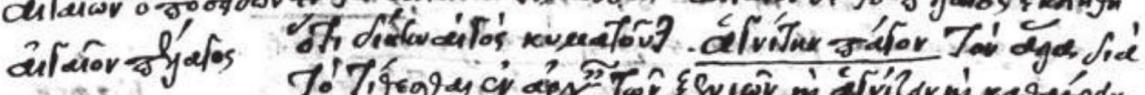

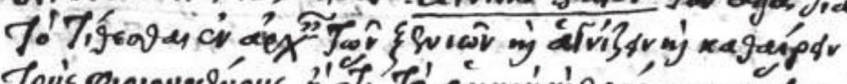

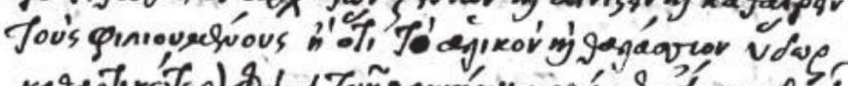

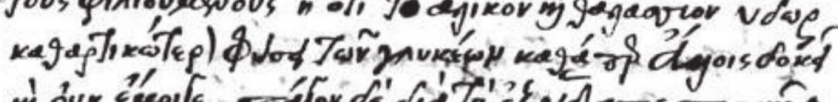
n' óve "real

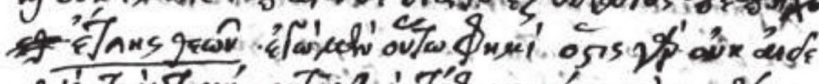

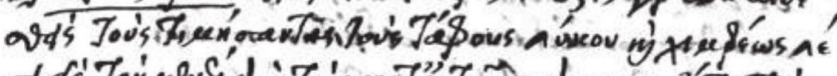

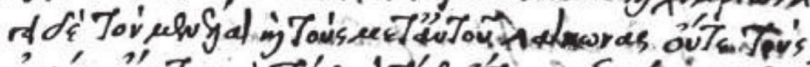

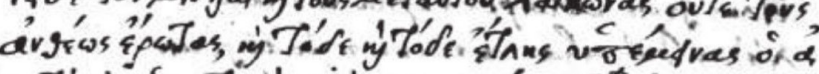

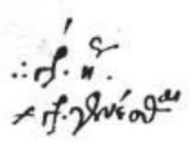

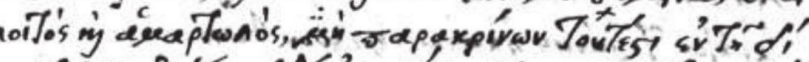

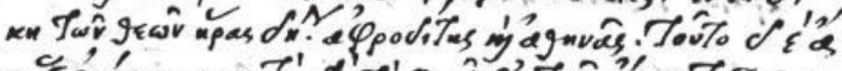

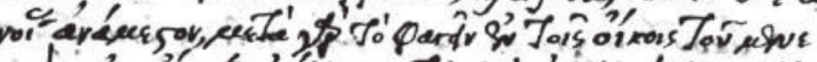

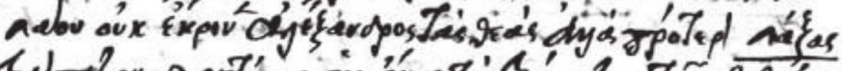

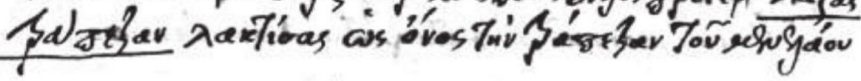

Figura 5. Salamanca ms. 9, f. 24v: posible muestra de la letra de Ducas, según Teresa Martínez Manzano, de la Universidad de Salamanca. 


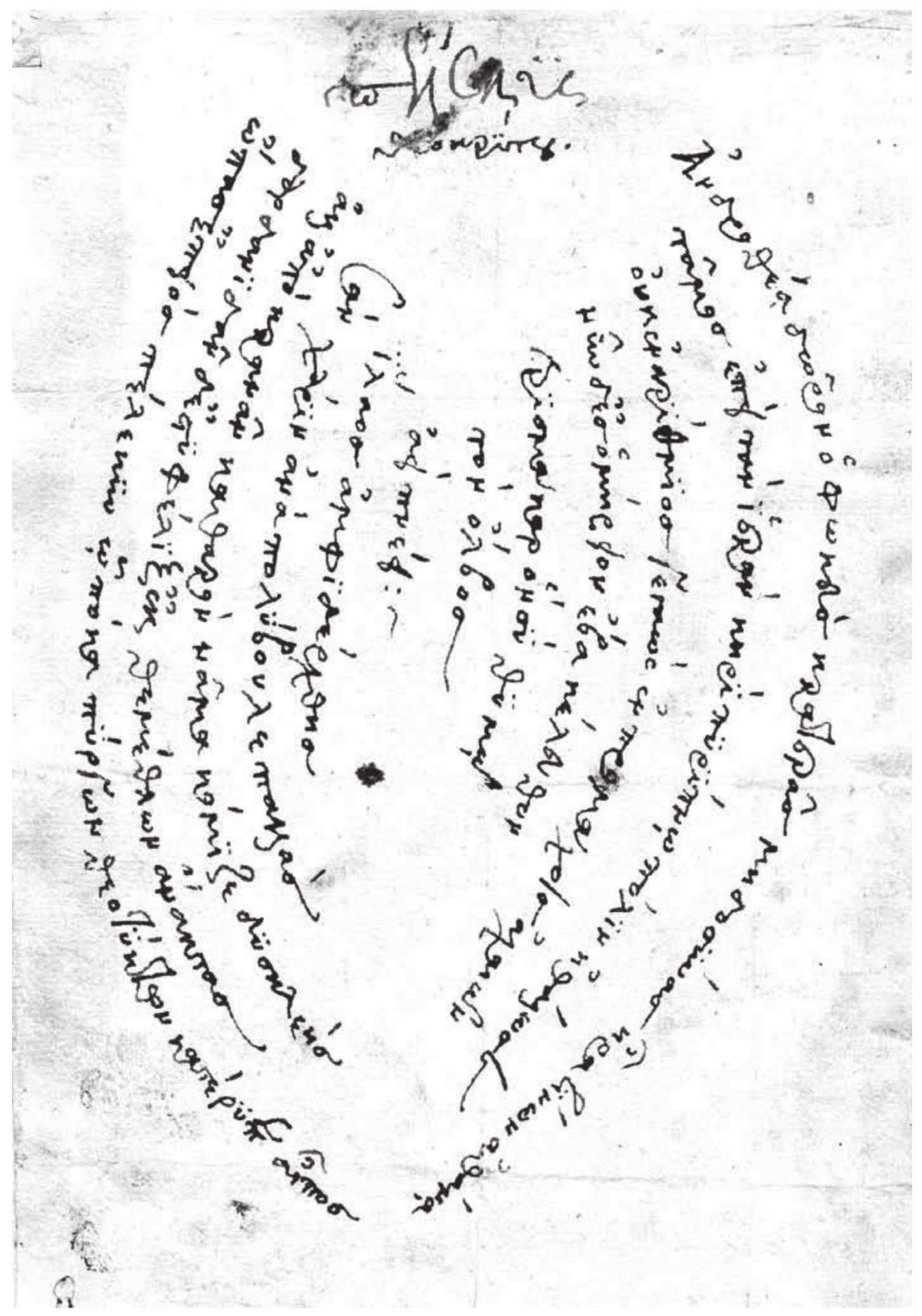

Figura 6. Salamanca, ms. 295, f. 120v, carmen figuratum ("hacha") debido probablemente a Demetrio Ducas, según identificación de Teresa Martínez Manzano. 